The estimated errors (chiefly from flight data for the aircraft path) are discussed at length. Four clocks were used on each journey, and delicate adjustments to the raw data were needed to correct for the vagaries of the clocks. For example, no two caesium clocks keep precisely the same time, but show systematic rate differences. Further, the rate of a clock may suddenly change, more or less permanently.

The agreement between theory and experiment is most satisfactory. As the authors conclude, "there seems to be little basis for further arguments about whether clocks will indicate the same time after a round trip, for we find that they do not".-W.B.

\section{COMPOSITE MATERIALS}

\section{Toughened Glass}

from our Materials Science Correspondent IT is one of nature's little ironies that many materials of particularly high strength are of little use as constructional materials. Substances such as silica, hard glass, alumina and boron nitride have extremely strong chemical bonding and thus very high intrinsic strength as well as high softening temperatures, but are also so brittle that they cannot safely be used under large loads, except in pure compression; the risk of fracture by mechanical or thermal shock is unacceptably great. There are promising exceptionsnotably silicon nitride (see Nature, 238, 128 ; 1972)-but glass and ceramics have usually been disappointing as materials for the mechanical engineer. This is why recent attempts to toughen these materials are specially important.

Reinforcement of weak materials with strong and stiff fibres or whiskers is now well understood in the laboratory and engineers' wariness is gradually diminishing. In industrial practice, all such composites have to date been based on polymeric matrices, most work having been carried out on glass-fibre reinforced epoxy resin. Carbon fibre is also being used to reinforce resins, but carbon-fibre reinforcement of metals for high-temperature use is less promising because of chemical attack of the fibres.

The very recent development of a radically new way of making continuous monofilaments of sapphire (J. T. A. Pollock, J. Mater. Sci., 7, 631, 649, 787; 1972; G. F. Hurley, ibid., 471) offers new prospects of making effective high-temperature composites based on metal matrices. Here the high strength of the filaments will simply be utilized and the metal matrix will serve merely as a binder to transfer stress to the fibres and (because of its deformability) to inhibit the spread of cracks

A rival strategy is to use a strong brittle matrix with strong brittle fibres, and to depend on the interaction between these two brittle constituents to generate toughness. This is the idea underlying recent work at Harwell on the reinforcement by carbon fibres of glass, alumina, magnesia and glass-ceramic (R. A. J. Sambell et al., ibid., 663, 676). Sambell and his colleagues prepared their materials by hot pressing, with either discontinuous fibres or aligned continuous fibres, up to 40 per cent volume fraction. Substantial increases in bend-strength were recorded with the continuous fibres but there were variable results with discontinuous fibres. The fracture toughness, however, increases for all matrices and all fibre arrangements; in the most favourable combinations the increase in the work of fracture was more than a thousand-fold. For discontinuous fibres, the work of fracture increased with fibre content up to 30 per cent; beyond this, no further benefit was normally obtained. For continuous fibres, however, a sharp increase in work of fracture came at about 50 per cent volume fraction, provided the method of pressing used was designed to prevent excessive damage to the fibres. The results were best for glass matrices, least good for magnesia.

Sambell et al. discuss their very promising findings in terms, especially, of the mismatch of thermal expansion coefficients of matrix and fibres; this mismatch leads to internal stresses and for some matrices, especially magnesia, leads to matrix cracking during cooling from the hot-pressing temperature, and such materials have relatively poor mechanical properties. Quite generally, however, matrix cracking occurred well before the ultimate tensile strength of the composite was reached.

Sambell et al. assume that the very high fracture toughness of some of their materials stems entirely from the work required to pull broken fibres out of the matrix, as cracks spread through the matrix. They ignore the work of fracture of the matrix itself, even though this must be far from negligible if the matrix is subject to multiple cracking. Sambell's analysis is cast in doubt by some work by G. A. Cooper and J. M. Sillwood of the National Physical Laboratory (ibid., 325).

Cooper and Sillwood studied a model system consisting of epoxy resin reinforced with regularly spaced continuous steel wires of various diameters and volume fractions. The matrix was caused to crack in a very regular pattern by pre-cooling the composite in liquid nitrogen. This technique was adopted for simplicity in preference to mechanical testing which, however, led to similar cracking. For most composite samples, the observed crack spacings so created varied with fibre radius and volume fraction $V_{\mathrm{f}}$ in a highly regular way: the crack spacing was proportional to $\left(1-V_{\mathrm{f}}\right) / V_{\mathrm{f}}$, and this is readily rationalized on the hypothesis that the critical fibre load-transfer length determines the crack spacing.

The really interesting feature, however, was the behaviour of samples with a high volume fraction of very fine wires. Such composites could not be cracked ahead of final failure, by either thermal or mechanical treatment. In an effort to understand this, Cooper

\title{
Different Haemoglobin Messengers
}

KAZAZIAN, in next Wednesday's Nature New Biology (August 9), shows that the rabbit haemoglobin messengers for $\alpha$ and $\beta$-chains differ in size, and he has separated and identified the two species. In polyacrylamide gel electrophoresis, the $10 \mathrm{~S}$ messenger RNA fraction gives rise to two bands of comparable intensity, which, it was surmised, were the two messengers. To verify this proposition, Kazazian introduced O-methylthreonine into the reticulocyte system. $\mathrm{O}$-methylthreonine is an analogue of isoleucine, which blocks incorporation of this amino-acid into the haemoglobin chains. The disturbance results in the formation of large polysomes containing mainly nascent $\beta$-chains, whereas the $\alpha$-chains, which contain isoleucine early in the sequence, are confined mostly to small polysomes.

Separation of these fractions and extraction of the messenger from each revealed that the component of lower electrophoretic mobility was reinforced in preparation from $\beta$-chain polysomes, and the higher mobility species in those from $\alpha$-chain polysomes. The difference in mobility seems to be truly related to molecular weight, for it persists when the base pairing is destroyed by formaldehyde treatment.

The calculated corresponding difference in size is somewhat bigger than would be expected in terms of the 5 per cent or so difference in the molecular weights of the $\alpha$ - and $\beta$-chains. Two bands are also observed in reticulocyte systems of other species. Kazazian has confirmed the result by using haemoglobin messenger from anaemic sheep of a genetic variant that synthesizes a third kind of chain in these conditions. This chain resembles the normal $\alpha$ rather than the $\beta$-chain in molecular weight. 